\title{
Formal Correctness Proof for DPLL Procedure*
}

\author{
Filip MARIĆ, Predrag JANIČIĆ \\ Faculty of Mathematics, University of Belgrade \\ Studentski trg 16, Belgrade, Serbia \\ e-mail: filip@matf.bg.ac.rs; janicic@matf.bg.ac.rs
}

Received: April 2008; accepted: January 2009

\begin{abstract}
The DPLL procedure for the SAT problem is one of the fundamental algorithms in computer science, with many applications in a range of domains, including software and hardware verification. Most of the modern SAT solvers are based on this procedure, extending it with different heuristics. In this paper we present a formal proof that the DPLL procedure is correct. As far as we know, this is the first such proof. The proof was formalized within the Isabelle/Isar proof assistant system. This proof adds to the growing body of formalized mathematical knowledge and it also provides a number of lemmas relevant for proving correctness of modern SAT and SMT solvers.
\end{abstract}

Keywords: SAT problem, DPLL procedure, formal proofs, Isabelle, Isar.

\section{Introduction}

The propositional satisfiability problem (SAT) is the problem of deciding whether there is a truth assignment under which a given propositional formula (in conjunctive normal form) evaluates to true. It is a canonical NP-complete problem (Cook, 1971) and it holds a central position in the field of computational complexity.

One of the first algorithms for testing satisfiability is a branch and backtracking procedure called Davis-Putnam-Logemann-Loveland (in short DPLL) procedure (Davis and Putnam, 1960; Davis et al., 1962). Although there have been many improvements to this procedure (including techniques called backjumping, conflict-driven lemma learning, restarts, etc.) it is still a core of the majority of the state-of-the-art complete SAT solvers (e.g., zChaff (Moskewicz et al., 2001), MiniSAT (Eén and Sörensson, 2003) and their successors). Modern SAT solvers show excellent performance even for huge formulae. They are used in many practical applications (electronic design automation, hardware and software verification, scheduling, etc.). SAT solving and modifications of the DPLL procedure such as DPLL(T) Nieuwnhuis et al. (2006) are also used for SMT (Satisfiability Modulo Theories) - the problem of deciding whether a given formula is satisfiable with respect to a background first-order theory $T$ (Ranise and Tinelli, 2006). SMT solving also has many important industrial applications. For a survey of SAT solvers, their performances, and applications see, for instance, Gu et al. (1997), Zhang and Malik (2002), Le Berre and Simon (2005).

\footnotetext{
${ }^{*}$ This work was partially supported by Serbian Ministry of Science grant 144030 .
} 
Despite their significance and wide applications, there are still no formal correctness proofs for SAT and SMT solvers, neither for the original DPLL procedure nor for its modern successors. For most of the modern SAT solvers, there are no even informal correctness proofs. In this paper we address this issue and present a first formalized correctness proof for the DPLL procedure, a proof that can be verified by an independent and reliable proof checking system. In its forty-five years history, there were no doubts about the correctness of this algorithm, but we believe that it is important to have its correctness proof formalized for, at least, the following two reasons: first, the correctness proof for the DPLL procedure will be useful for checking correctness of modern SAT and SMT solvers, some of which are still unreliable ${ }^{1}$; second, this proof adds to the growing body of formalized, verifiable mathematical knowledge (which is important as the rigour, reliability, and objectivity of formal proofs is vital in many computer science applications, such as software and hardware verification).

Our correctness proof for the DPLL procedure is formalized within Isabelle proof assistant and for object-level proofs we use Isar (Intelligible semi-automated reasoning) language, natively supported in Isabelle. For definitions of some functions we use primitive recursion, also natively supported in Isabelle. We also use Isabelle's built-in theory of lists (Nipkow et al., 2005; p. 16) and, to a limited extent, Isabelle's built-in theory of sets (only for finite sets) (Nipkow et al., 2005; p. 109).

Overview of the paper. In Section 2 we give background information on the DPLL algorithm, on formal proofs and the Isabelle/Isar system, and on program verification. In Section 3 we give basic notation, definitions and properties of propositional logic, required for our proof. In Section 4 we give a formalization of the DPLL procedure (with one concrete implementation discussed in Appendix), and in Section 5 we prove the procedure's total correctness (partial correctness and termination). In Section 6, we discuss some technical details and give some fragments of our formalization made in Isabelle/Isar. In Section 7 we briefly discuss related work, and in Section 8 we draw final conclusions and discuss future work.

\section{Background}

\subsection{Davis-Putnam-Logemann-Loveland or DPLL algorithm}

The Davis-Putnam procedure was introduced in 1960 by Martin Davis and Hilary Putnam. Two years later, Martin Davis, George Logemann, and Donald W. Loveland introduced a refined version of the algorithm, in which they replaced the elimination rule by a splitting rule (Davis et al., 1962). In this newer version, the splitting rule leads to two smaller subproblems (one for each truth value for a selected variable), instead of a single, possibly larger, subproblem generated by the elimination rule. Nowadays, this later version of the algorithm is often referred to as DPLL procedure. The algorithm is shown in Fig. 1. In the algorithm, $\Phi$ is a set of propositional clauses tested for satisfiability. $\Phi(l)$

\footnotetext{
${ }^{1}$ For instance, over the previous years, several SMT solvers turned out to be unsound according to the results from SMT competitions, http: / / www. smtcomp. org/.
} 


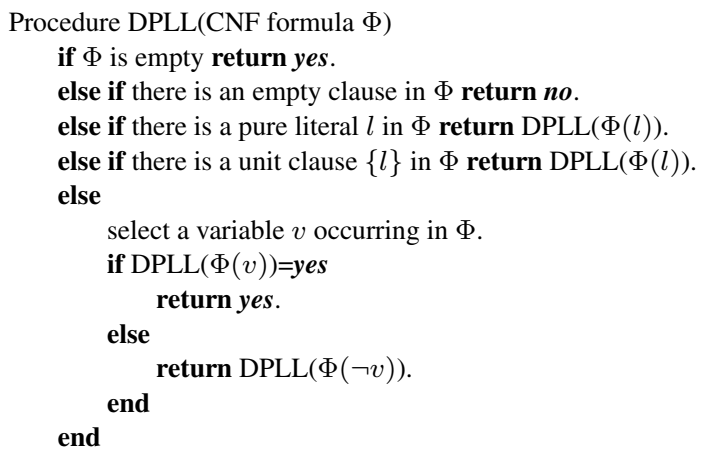

Fig. 1. DPLL procedure.

denotes the formula obtained from $\Phi$ by substituting a literal $l$ by $\top$, by substituting the opposite literal of $l$ by $\perp$, and by simplifying afterwards. A literal is pure if it occurs in the formula but its opposite literal does not occur. A clause is unit if it contains only one literal. A non-recursive version of the algorithm can be found in Davis et al. (1994). There are also rule based descriptions of some more advanced versions of this algorithm (Krstić and Goel, 2007; Nieuwnhuis et al., 2006).

The selection of a variable $v$ within the given algorithm is critical for its performance. Choosing a variable may be trivial - choosing a first remaining variable or a random variable, but it can also be very complex. In the original version of the procedure, the variable occurring in the first clause of minimal length was chosen. The worst case complexity for this procedure on 3-SAT (3-SAT is a variant of the SAT problem, with all clauses consisting of exactly three literals) is $O\left(2^{0.762 n}\right)$ (Cook and Mitchell, 1997). For more references on selecting a split variable and on worst case complexity analysis of the DPLL procedure see, for instance, Cook and Mitchell (1997), Irgens and Havens (2004).

\subsection{Formal proofs and Isabelle}

Over the last years, in all areas of mathematics and computer science, with a history of huge number of flawed published mathematical proofs and also flawed software and hardware components, formal proofs (machine verifiable, given in object-level form, in terms of axioms and inference rules) have gained more and more importance. There are growing efforts in this direction, with many extremely complex mathematical theorems formally proved ${ }^{2}$ and with many software tools producing and checking formal proofs. Isabelle is a generic theorem prover that supports a variety of logics, with Gentzen's natural deduction as the basic built-in logic (Paulson, 1994). Distinctive Isabelle's features include representation of logics within a meta-logic and the use of higher-order unification to combine inference rules. Isabelle can be applied to reasoning in pure mathematics or verification of computer systems. Isabelle is one of the most popular theorem proving systems nowadays.

\footnotetext{
${ }^{2}$ For a list of selected formally proved theorems see, for instance, http : / / www . cs.ru . nl / freek/ $100 \%$.
} 


\subsection{Readable formal proofs and Isar}

Theorem proving system supporting both interactive proof development and some degree of automation have become quite successful in sizable applications in recent years. Most of them are based on traditional proof scripts which explicitly list all axioms and inference rules used in every single proof step. Despite success of semi-automated proving systems based on such scripts in formalizing fragments of mathematics and computer science, they are still not accepted by a wide range of researchers. The Intelligible semiautomated reasoning (Isar; Wenzel, 2007) approach to readable formal proof documents aims to bridge the semantic gap between internal notions of proof given by state-ofthe-art interactive theorem proving systems and an appropriate level of abstraction for user-level work. Isar is an alternative proof language interface layer, beyond traditional formal proof tactic scripts, which is much more readable for the users. The Isabelle/Isar system provides an interpreter for the Isar formal proof document language, and readable Isar proof documents are converted and executed as series of low-level inference steps. It allows users to express proofs in a human-friendly way but still have proofs that are automatically formally verified by an underlying proof system and that rely only on valid axioms and inference rules.

\subsection{Program verification}

Program verification is the process of formally proving that a computer program meets its specification. Program verification is old, but very much active field. Following the lessons from major software failures in recent years, more and more efforts have been invested in this field. Many fundamental algorithms and properties of data structures have been formalized. Also, a lot of work has been devoted to formalization of compilers, program semantics, communication protocols, security protocols, etc. Formal verification is vital for SAT and SMT solvers and first steps in this direction have been made. For a short overview of results in program verification see Section 7.

\section{Notation and Definitions}

In this section, we introduce notation, definitions, and basic propositions used in our formalized correctness proof for the DPLL procedure. Our proof is almost self-contained, so here we also define notions (and notation) of literals, clauses, formulae, satisfiability, etc. All notions introduced here are also formalized within Isabelle's higher order logic (Isabelle/HOL). Some of them are defined by primitive recursion, supported in Isabelle/HOL.

Formulae and logical connectives of this meta-logic $(\wedge, \vee, \neg, \Rightarrow, \Leftrightarrow)$ are written in the usual way. Ternary $i f$-then else connective is also used: if $f$ then $f_{1}$ else $f_{2}$ denotes $f \Rightarrow f_{1} \wedge \neg f \Rightarrow f_{2}$. The symbol $=$ denotes syntactical identity of two expressions. ${ }^{3}$

\footnotetext{
${ }^{3}$ Note that in this presentation we make a clear distinction between syntactical identity and logical equivalence and use different symbols for them, $=$ and $\Leftrightarrow$ (while in Isabelle/HOL these two notions are denoted by the same symbol, $=$ ).
} 


\begin{tabular}{|c|c|}
\hline[] & The empty list. \\
\hline$\left[e_{1}, \ldots, e_{n}\right]$ & The list of $n$ given elements $e_{1}, \ldots, e_{n}$. \\
\hline$e \in$ list & $e$ is a member of the list list. \\
\hline e\# list & The list obtained by prepending element $e$ to the list list. \\
\hline list $_{1} @$ list $_{2}$ & The list obtained by appending lists list $_{1}$ and list $_{2}$. \\
\hline head(list) & The first element of the list list (assuming the list is nonempty). \\
\hline tail(list) & The list obtained by removing the first element of the list list. \\
\hline list \e & $\begin{array}{l}\text { The list obtained by removing all occurrences of the element } \\
e \text { from the list list. }\end{array}$ \\
\hline list $_{1} \backslash$ list $_{2}$ & $\begin{array}{l}\text { The list obtained from the list } \text { list }_{1} \text { by removing from it } \\
\text { all elements of the list } \text { list }_{2} \text {. }\end{array}$ \\
\hline list $_{1} \subseteq$ list $_{2}$ & All elements of list $_{1}$ are also elements of list $_{2}$. \\
\hline $\mid$ list $\mid$ & The length of the list list. \\
\hline\{\} & The empty set. \\
\hline$e \in$ set & $e$ is a member of the set set. \\
\hline $\operatorname{set}_{1} \cup \operatorname{set}_{2}$ & The union of the sets set $_{1}$ and set $_{2}$. \\
\hline
\end{tabular}

Fig. 2. Notions from the theory of lists and the theory of sets that are used.

The theory that we built for expressing correctness of the DPLL procedure uses Isabelle's built-in theory of lists and Isabelle's built-in theory of sets (only for finite sets). Fig. 2 informally describes the notions from these theories that we use.

We assume that all meta-logic formulae in the following text are implicitly universally quantified, if not stated otherwise. We use typed logic, but for better readability, when printing formulae we omit types, and use the following convention:

- $v b l$ denotes a variable and has the type nat;

- $l, l^{\prime}, l_{1}, l_{2}, \ldots$ denote literals and have the type Literal;

- $c, c^{\prime}, c_{1}, c_{2}, \ldots$ denote clauses and have the type Clause;

- $F, F^{\prime}, F_{1}, F_{2}, \ldots$ denote formulae and have the type Formula;

- $v, v^{\prime}, v_{1}, v_{2}, \ldots$ denote valuations and have the type Valuation.

DEFINITION 1. A variable is identified with a natural number.

DEFINITION 2. A literal is either a positive variable (denoted by $+v b l$ ) or a negative variable (denoted by $-v b l$ ).

DEFINITION 3. A clause is a list of literals.

Definition 4. A formula is a list of clauses.

Definition 5. A valuation is a list of literals.

DEFINITION 6. A variable of a literal, denoted $\operatorname{var}(l)$, is defined in the following way: $\operatorname{var}(+v b l)=\operatorname{var}(-v b l)=v b l$.

DEFINITION 7. A opposite literal of a literal, denoted $\bar{l}$, is defined in the following way: $\overline{+v b l}=-v b l, \overline{-v b l}=+v b l$. 
Notice that we abuse the notation and overload some symbols. For example, the symbol $\in$ denotes both set membership and list membership. It is also used to denote that a literal occurs in a formula.

DEFINITION 8. A formula $F$ contains a literal $l$ (and the literal $l$ occurs in the formula $F)$, denoted $l \in F$, iff $(\exists c)(c \in F \wedge l \in c)$.

Symbol vars is also overloaded and denotes the set of variables occurring in a clause, formula, valuation, defined by primitive recursion.

DEFINITION 9. A set of variables that occur in a clause $c$, denoted vars $(c)$, is defined in the following way:

$$
\begin{aligned}
& \operatorname{vars}([])=\{\} \\
& \operatorname{vars}(l \# c)=\operatorname{var}(l) \cup \operatorname{var} s(c)
\end{aligned}
$$

A set of variables that occur in a formula $F$, denoted $\operatorname{var} s(F)$, is defined in the following way:

$$
\begin{aligned}
& \operatorname{vars}([])=\{\} \\
& \operatorname{vars}(c \# F)=\operatorname{vars}(c) \cup \operatorname{vars}(F)
\end{aligned}
$$

A set of variables that occur in a valuation $v$, denoted $\operatorname{var} s(v)$, is defined in the following way:

$$
\begin{aligned}
& \operatorname{vars}([])=\{\} \\
& \operatorname{vars}(l \# v)=\operatorname{var}(l) \cup \operatorname{vars}(v)
\end{aligned}
$$

The semantics is introduced by the following definitions.

Definition 10. A literal $l$ is true in a valuation $v$, denoted $v \vDash l$, iff $l \in v$.

A clause $c$ is true in a valuation $v$, denoted $v \vDash c$, iff $(\exists l)(l \in c \wedge v \vDash l)$.

A formula $F$ is true in a valuation $v$, denoted $v \vDash F$, iff $(\forall c)(c \in F \Rightarrow v \vDash c)$.

DEFINITION 11. A literal $l$ is false in a valuation $v$, denoted $v \vDash \neg l$, iff $\bar{l} \in v$.

A clause $c$ is false in a valuation $v$, denoted $v \vDash \neg c$, iff $(\forall l)(l \in c \Rightarrow v \vDash \neg l)$.

A formula $F$ is false in a valuation $v$, denoted $v \vDash \neg F$, iff $(\exists c)(c \in F \wedge v \models \neg c)$.

Definition 12. A valuation $v$ is inconsistent iff it contains both literal and its opposite i.e., $(\exists l)(v \vDash l \wedge v \vDash \bar{l})$. A valuation is consistent iff it is not inconsistent.

DEFINITION 13. A model of a formula $F$ is a consistent valuation $v$ under which $F$ is true i.e., $\operatorname{model}(v, F)$ iff consistent $(v) \wedge v \vDash F$. A formula $F$ is satisfiable, denoted $\operatorname{sat}(F)$ iff it has a model i.e., $(\exists v)(\operatorname{model}(v, F))$ 
The following proposition gives some basic properties of the notions we have just introduced. These properties were formulated and proved in Isabelle/Isar and used in the correctness proof for the DPLL procedure.

\section{PROPOSITION 1}

\begin{tabular}{|c|c|}
\hline & Basic properties of opposite literals: \\
\hline $\begin{array}{l}(A 1) \\
(A 2) \\
(A 3) \\
(A 4) \\
(A 5) \\
(A 6) \\
\end{array}$ & $\begin{array}{l}\overline{\bar{l}}=l \\
\overline{l_{1}}=l_{2} \Leftrightarrow \overline{l_{2}}=l_{1} \\
\overline{l_{1}}=\overline{l_{2}} \Leftrightarrow l_{1}=l_{2} \\
\bar{l} \neq l \\
\operatorname{var}(\bar{l})=\operatorname{var}(l) \\
\operatorname{var}\left(l_{1}\right)=\operatorname{var}\left(l_{2}\right) \Leftrightarrow l_{1}=l_{2} \vee l_{1}=\overline{l_{2}}\end{array}$ \\
\hline $\begin{array}{l}(B 1) \\
(B 2) \\
(B 3) \\
(B 4) \\
(B 5) \\
(B 6) \\
(B 7) \\
\end{array}$ & $\begin{array}{l}\text { Basic properties of } \operatorname{vars:} \\
l \in c \Rightarrow \operatorname{var}(l) \in \operatorname{var} s(c) \\
l \in F \Rightarrow \operatorname{var}(l) \in \operatorname{vars}(F) \\
c \in F \Rightarrow \operatorname{var}(c) \subseteq \operatorname{vars}(F) \\
l \in v \Rightarrow \operatorname{var}(l) \in \operatorname{vars}(v) \\
\operatorname{var}(l) \in \operatorname{vars}(c) \Leftrightarrow l \in c \vee \bar{l} \in c \\
\operatorname{var}(l) \in \operatorname{var}(F) \Leftrightarrow l \in F \vee \bar{l} \in F \\
\operatorname{var}(l) \in \operatorname{vars}(v) \Leftrightarrow v \vDash l \vee v \vDash \neg l\end{array}$ \\
\hline $\begin{array}{l}(C 1) \\
(C 2) \\
\end{array}$ & $\begin{array}{l}\text { Basic properties of consistent valuations: } \\
\text { consistent }([]) \\
\text { inconsistent }(v \backslash l) \Rightarrow \text { inconsistent }(v)\end{array}$ \\
\hline $\begin{array}{l}(D 1) \\
(D 2)\end{array}$ & $\begin{array}{l}\text { Basic properties of the relation } \vDash \text { : } \\
v \vDash c \backslash l \Rightarrow v \vDash c \\
\operatorname{var}(l) \notin \operatorname{vars}(F) \wedge v \vDash F \Rightarrow v \backslash[l, \bar{l}] \vDash F\end{array}$ \\
\hline $\begin{array}{l}(E 2) \\
(E 3) \\
(E 4) \\
\end{array}$ & $\begin{array}{l}\text { Basic properties of models and formula satisfiability: } \\
\text { model }(v, F) \wedge \operatorname{vbl} \notin \operatorname{vars}(F) \Rightarrow \\
\quad\left(\exists v^{\prime}\right)\left(\operatorname{model}\left(v^{\prime}, F\right) \wedge \operatorname{vbl} \notin \operatorname{vars}\left(v^{\prime}\right)\right) \\
F \subseteq F^{\prime} \Rightarrow\left(\operatorname{sat}\left(F^{\prime}\right) \Rightarrow \operatorname{sat}(F)\right) \\
\operatorname{sat}([]) \\
{[] \in F \Rightarrow \neg \operatorname{sat}(F)}\end{array}$ \\
\hline
\end{tabular}

\section{Formalization of the DPLL Procedure}

In this section, we present a formalization of the DPLL procedure and all required notions. We give a special attention to the notions of pure literal and unit clause, essential for certain steps of the procedure. All the given proofs are rigorously formulated and verified within Isabelle/Isar.

\subsection{Substitution}

One of the basic steps of the DPLL procedure is substitution of a literal by logical constants $\top$ and $\perp$, and simplification of the obtained formula. This operation is formalized by the following definition. 
DEFINITION 14. $F[l \rightarrow T]$ is the formula that is obtained from $F$ by deleting all clauses that contain $l$ and deleting all occurences of the literal $\bar{l}$. It is defined by primitive recursion:

$$
\begin{aligned}
{[][l \rightarrow \top]=} & {[] } \\
(c \# F)[l \rightarrow \top]= & \text { if } l \in c \text { then } \\
& F[l \rightarrow \top] \\
& \text { else if } \bar{l} \in c \text { then } \\
& \quad(c \backslash \bar{l}) \# F[l \rightarrow \top] \\
& \text { else } \quad \\
& c \# F[l \rightarrow \top]
\end{aligned}
$$

$F[l \rightarrow \perp]$ denotes $F[\bar{l} \rightarrow \top]$

The following proposition (proved in Isabelle/Isar) gives some basic properties of this operation.

\section{Proposition 2.}

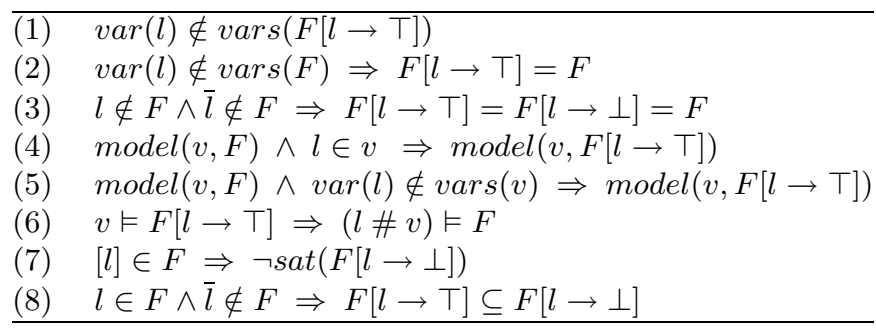

The following lemma suggests that the satisfiability of a formula can be, by using substitution, checked by testing the satisfiability of two smaller formulae. Since this is a fundamental lemma in the proof of the DPLL correctness, we give a sketch of its proof. This sketch is still very close to its formal, Isabelle/Isar counterpart. It also illustrates the use of the listed properties.

Lemma 1 (split rule lemma).

$$
\operatorname{sat}(F) \Leftrightarrow \operatorname{sat}(F[l \rightarrow \top]) \vee \operatorname{sat}(F[l \rightarrow \perp])
$$

Proof.

$(\Rightarrow)$ : Let us assume $\operatorname{sat}(F)$. This means that there is a valuation $v$ such that $\operatorname{model}(v, F)$, i.e., consistent $(v)$ and $v \vDash F$. We consider two cases:

1. $\operatorname{var}(l) \notin \operatorname{vars}(v)$ : from Proposition 2(5) it follows that $v$ is a model for $F[l \rightarrow \top]$, and, therefore, it holds that $\operatorname{sat}(F[l \rightarrow \top])$.

2. $\operatorname{var}(l) \in \operatorname{vars}(v)$ : from Proposition 1(B7), either $v \vDash l$ or $v \vDash \neg l$ holds.

(a) $v \vDash l$ : from Proposition 2(4) it follows that $v$ is a model for $F[l \rightarrow \top]$, and, therefore, it holds that $\operatorname{sat}(F[l \rightarrow \top])$. 
(b) $v \vDash \bar{l}$ : from Proposition 2(4), it holds that $v$ is a model for $F[\bar{l} \rightarrow \top]$, and, therefore, it holds that $\operatorname{sat}(F[l \rightarrow \perp])$.

$(\Leftarrow)$ : Let us assume $\operatorname{sat}(F[l \rightarrow \top]) \vee \operatorname{sat}(F[l \rightarrow \perp])$.

Consider the case when $\operatorname{sat}(F[l \rightarrow T])$ holds. This means that there is a valuation $v$ such that $\operatorname{model}(v, F)$, i.e., $\operatorname{consistent}(v)$ and $v \vDash F[l \rightarrow \top]$. From Proposition 2(1), it holds that $\operatorname{var}(l) \notin \operatorname{vars}(F[l \rightarrow \top])$. From Proposition 1(E1) applied to the formula $F[l \rightarrow \top]$, variable $\operatorname{var}(l)$, and the valuation $v$, it follows that there is a valuation $v^{\prime}$ such that consistent $\left(v^{\prime}\right), v^{\prime} \vDash F[l \rightarrow \top]$ and $\operatorname{var}(l) \notin \operatorname{vars}\left(v^{\prime}\right)$. Then, from Proposition 2(6) applied to the valuation $v^{\prime}$, it follows that $\left(l \# v^{\prime}\right) \vDash F$. Since $\operatorname{var}(l) \notin \operatorname{vars}\left(v^{\prime}\right)$, it follows $\bar{l} \notin v^{\prime}$ and therefore consistent $\left(l \# v^{\prime}\right)$. Finally, $\operatorname{sat}(F)$ holds since $l \# v^{\prime}$ is a model of $F$.

The case when $\operatorname{sat}(F[l \rightarrow \perp])$ holds is analogous to the previous case, with the literal $l$ replaced by $\bar{l}$.

This lemma inspires a naive, but still sound and complete, procedure for satisfiability checking. In some situations, one of the two formulae $\operatorname{sat}(F[l \rightarrow \top])$ and $\operatorname{sat}(F[l \rightarrow \perp])$ from the above lemma does not need to be considered. For instance, if the first disjunct is satisfied, then the second one does not need to be checked (as indicated by the algorithm shown in Fig. 1). Also, in some special cases discussed below it suffices to consider just one of these disjuncts.

\subsection{Unit Clauses}

One sort of optimization of the mentioned naive procedure for satisfiability checking is based on exploiting unit clauses.

Definition 15. A clause $c$ is a unit clause iff it has only one literal, i.e., $c=[l]$. Then we also say that $l$ is a unit literal.

The following lemma shows that when a formula contains a unit clause, checking its satisfiability can be reduced to checking satisfiability of just one smaller formula (in constrast to Lemma 1).

Lemma 2 (unit clause rule lemma).

$$
[l] \in F \Rightarrow(\operatorname{sat}(F) \Leftrightarrow \operatorname{sat}(F[l \rightarrow \top]))
$$

Proof. By Proposition 2(7) it holds that $[l] \in F \Rightarrow \neg \operatorname{sat}(F[l \rightarrow \perp])$, so the lemma is a direct consequence of the split rule lemma (Lemma 1).

\subsection{Pure Literals}

Another sort of optimizations of the naive procedure for satisfiability checking is based on pure literals. 
Definition 16. A literal $l$ is a pure literal in $F$ iff $l \in F$ and $\bar{l} \notin F$.

Lemma 3 (pure literal rule lemma).

$$
l \in F \wedge \bar{l} \notin F \Rightarrow(\operatorname{sat}(F) \Leftrightarrow \operatorname{sat}(F[l \rightarrow \top]))
$$

Proof. By Proposition 2(8) it holds that

$$
l \in F \wedge \bar{l} \notin F \Rightarrow F[l \rightarrow \top] \subseteq F[l \rightarrow \perp],
$$

so the lemma is a simple consequence of the Proposition 1(E2) applied to $F[l \rightarrow T]$ and $F[l \rightarrow \perp]$, and the Split rule lemma (Lemma 1).

\subsection{Definition of the DPLL Procedure}

A recursive definition of the DPLL procedure is given in the following definition.

\section{DEFINITION 17 .}

$$
\begin{aligned}
& \operatorname{dpll}(F) \Leftrightarrow \\
& \text { if } F=[] \text { then } \\
& \begin{array}{c}
\top \\
\text { else if }[] \in F \text { then } \\
\perp
\end{array} \\
& \text { else if hasPureLiteral }(F) \text { then } \\
& \quad d p l l(F[\text { getPureLiteral }(F) \rightarrow \top]) \\
& \text { else if } \operatorname{has} U \text { nitLiteral }(F) \text { then } \\
& \quad d p l l(F[\text { getUnitLiteral }(F) \rightarrow \top]) \\
& \text { else if } \operatorname{dpll}(F[\text { selectLiteral }(F) \rightarrow \top] \text { then } \\
& \quad \begin{array}{l}
\text { else } \\
\text { dpll }(F[\text { selectLiteral }(F) \rightarrow \perp]
\end{array}
\end{aligned}
$$

Notice that the functions getUnitLiteral, getPureLiteral and selectLiteral returning literals and Boolean functions hasUnitLiteral, hasPureLiteral, must be effectively defined in order to have an effective DPLL procedure. As said in Section 2, this can be done in many ways. The choice of a specific implementation of these functions, can affect the procedure performance but does not affect its correctness, as long as they meet the following specification (their sorts are obvious from the context):

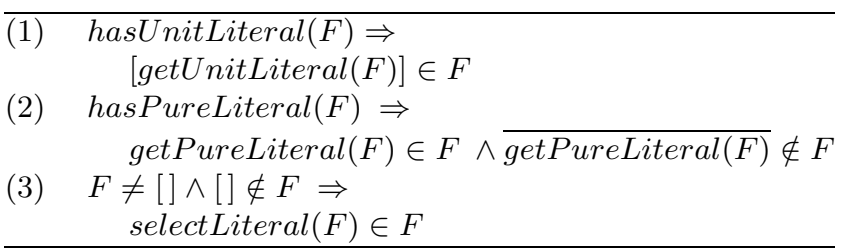

One simple way to define these functions is given in Appendix. 


\section{Termination and Correctness of the DPLL Procedure}

In this section we prove termination and, finally, correctness of the DPLL procedure. Our proof roughly follows the informal proof given in Davis et al. (1994). In Isabelle spirit, termination is ensured by defining a measure that is decreased by each recursive call of the procedure. This property is ensured by proving several propositions corresponding to different recursive calls.

\subsection{Termination}

In order to prove termination of the specified procedure, we show that the total number of literals in all clauses of $F$ is decreased by each recursive call. ${ }^{4}$ This number, denoted by numLiterals $(F)$, is defined by primitive recursion.

\section{DEFINITION 18.}

$$
\begin{aligned}
& \operatorname{numLiterals}([])=0 \\
& \operatorname{numLiterals}(c \# F)=|c|+\operatorname{numLiterals}(F)
\end{aligned}
$$

From the following proposition it follows that the total number of literals in $F$ is reduced by each recursive call. Because of that, the total number of literals in the formula can be used as a decreasing measure suitable for proving termination of the DPLL procedure. This measure and the following proposition are used by Isabelle/Isar for the automatic proof of termination.

\section{PROPOSITION 3 .}

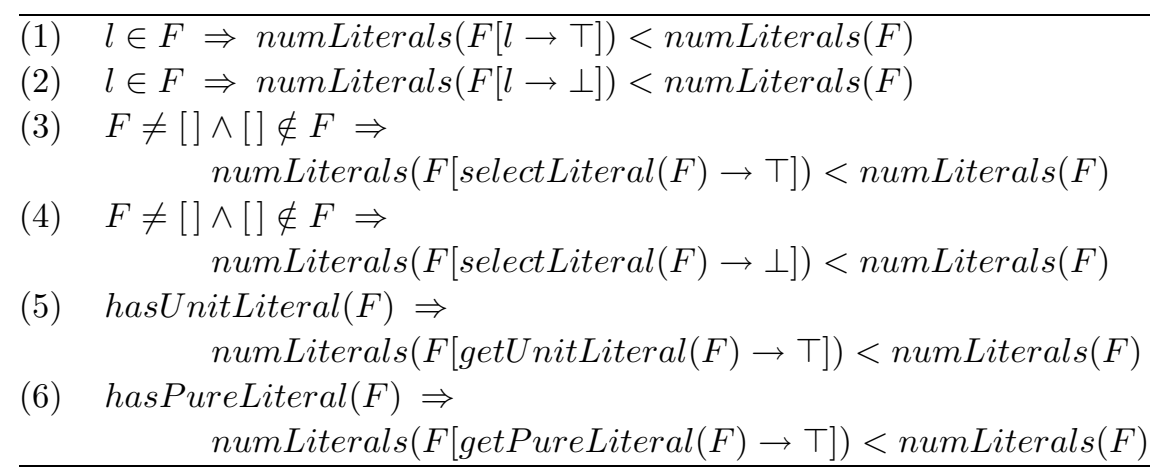

\subsection{Correctness}

Finally, we can prove the correctness of the procedure defined by Definition 17.

\footnotetext{
${ }^{4}$ There are other suitable termination measures that can be used as well (e.g., the number of occuring variables).
} 


\section{Theorem 1.}

$$
\operatorname{dpll}(F) \Leftrightarrow \operatorname{sat}(F)
$$

Proof. As a base of the inductive proof, we consider the cases in which the function does not perform a recursive call. There are two such branches:

- If $F=$ [] then, by Proposition 1(E3), $\operatorname{dpll}(F)=\top$ and $\operatorname{sat}(F)=\top$, so the conjecture trivially holds.

- If $F \neq[]$ and [] $\in F$ then, by Proposition 1(E4), $\operatorname{dpll}(F)=\perp$ and $\operatorname{sat}(F)=\perp$, so the conjecture trivially holds.

Now, let us assume that the conjecture holds for each recursive call, and let us show that the conjecture holds for the top level procedure call. Therefore, let us assume the following inductive hypotheses.

$$
\begin{aligned}
& (F \neq[] \wedge[] \notin F) \Rightarrow \\
& \text { (hasPureLiteral }(F) \Rightarrow \\
& \operatorname{dpll}(F[\operatorname{getPureLiteral}(F) \rightarrow \top]) \Leftrightarrow \operatorname{sat}(F[\operatorname{getPureLiteral}(F) \rightarrow \top])) \\
& (F \neq[] \wedge[] \notin F \wedge \neg \text { hasPureLiteral }(F)) \Rightarrow \\
& \text { (hasUnitLiteral }(F) \Rightarrow \\
& \operatorname{dpll}(F[\operatorname{getUnitLiteral}(F) \rightarrow \top]) \Leftrightarrow \operatorname{sat}(F[\operatorname{getUnitLiteral}(F) \rightarrow \top])) \\
& (F \neq[] \wedge[] \notin F \wedge \neg \text { hasPureLiteral }(F) \wedge \neg \text { hasUnitLiteral }(F)) \Rightarrow \\
& \operatorname{dpll}(F[\operatorname{selectLiteral}(F) \rightarrow \top]) \Leftrightarrow \operatorname{sat}(F[\operatorname{selectLiteral}(F) \rightarrow \top]) \\
& (F \neq[] \wedge[] \notin F \wedge \neg \text { hasPureLiteral }(F) \wedge \neg \text { hasUnitLiteral }(F)) \Rightarrow \\
& (\neg \operatorname{dpll}(F[\operatorname{selectLiteral}(F) \rightarrow \top]) \Rightarrow \\
& \operatorname{dpll}(F[\operatorname{selectLiteral}(F) \rightarrow \perp]) \Leftrightarrow \operatorname{sat}(F[\operatorname{selectLiteral}(F) \rightarrow \perp]))
\end{aligned}
$$

Let us consider different branches of $i f$-then-else in the definition of $d p l l$ function:

- If $F \neq[]$ and [] $\notin F$, and hasPureLiteral $(F)$, then by the dpll definition:

$$
\operatorname{dpll}(F) \Leftrightarrow \operatorname{dpll}(F[\operatorname{getPureLiteral}(F) \rightarrow \top])
$$

Also, by the inductive hypothesis, it holds:

$$
\operatorname{dpll}(F[\operatorname{getPureLiteral}(F) \rightarrow \top]) \Leftrightarrow \operatorname{sat}(F[\operatorname{getPureLiteral}(F) \rightarrow \top])
$$

From the specification of getPureLiteral and the assumption hasPureLiteral $(F)$, it holds that getPureLiteral $(F) \in F \wedge \overline{\text { getPureLiteral }(F)}$ $\notin F$. Then, by Lemma 3:

$$
\operatorname{sat}(F[\text { getPureLiteral }(F) \rightarrow \top]) \Leftrightarrow \operatorname{sat}(F)
$$


Therefore, $\operatorname{dpll}(F) \Leftrightarrow \operatorname{sat}(F)$.

- If $F \neq[]$ and [] $\notin F$ and $\neg$ hasPureLiteral $(F)$, and hasUnitLiteral $(F)$, then by the $d p l l$ definition:

$$
\operatorname{dpll}(F) \Leftrightarrow \operatorname{dpll}(F[\operatorname{get} U \operatorname{nitLiteral}(F) \rightarrow \top])
$$

Also, by the inductive hypothesis, it holds:

$$
\operatorname{dpll}(F[\operatorname{get} U n i t L i t e r a l(F) \rightarrow \top]) \Leftrightarrow \operatorname{sat}(F[\operatorname{get} U n i t L i t e r a l(F) \rightarrow \top])
$$

From the specification of getUnitLiteral and the assumption hasUnitLiteral $(F)$, it holds that $[$ getUnitLiteral $(F)] \in F$. Then, by Lemma 2:

$$
\operatorname{sat}(F[\operatorname{get} U n i t L i t e r a l(F) \rightarrow \top]) \Leftrightarrow \operatorname{sat}(F)
$$

Therefore, $\operatorname{dpll}(F) \Leftrightarrow \operatorname{sat}(F)$.

- If $F \neq[]$ and [] $\notin F$ and $\neg$ hasPureLiteral $(F)$, and $\neg$ hasUnitLiteral $(F)$ then, from the specification of $d p l l$ and the definition of if - then - else connective, it holds that

$$
\begin{aligned}
& \operatorname{dpll}(F) \Leftrightarrow(d p l l(F[\operatorname{selectLiteral}(F) \rightarrow \top]) \Rightarrow \top) \wedge \\
&(\neg d p l l(F[\operatorname{selectLiteral}(F) \rightarrow \top]) \Rightarrow \\
& d \operatorname{pll}(F[\operatorname{selectLiteral}(F) \rightarrow \perp])
\end{aligned}
$$

Therefore, it holds that

$$
\begin{aligned}
d p l l(F) \Leftrightarrow & d p l l(F[\operatorname{selectLiteral}(F) \rightarrow \top]) \vee \\
& d p l l(F[\text { selectLiteral }(F) \rightarrow \perp]) .
\end{aligned}
$$

If $\operatorname{dpll}(F[\operatorname{select} \operatorname{Literal}(F) \rightarrow \top])$ then, by the inductive hypothesis, it holds that $\operatorname{sat}(F[\operatorname{selectLiteral}(F) \rightarrow \top])$. Otherwise, if $\neg \operatorname{dpll}(F[\operatorname{select} \operatorname{Literal}(F) \rightarrow$ $\top])$ and $\operatorname{dpll}(F[\operatorname{selectLiteral}(F) \rightarrow \perp])$ hold then, by the inductive hypothesis, $\operatorname{sat}(F[\operatorname{select} \operatorname{Literal}(F) \rightarrow \perp])$ holds. Therefore:

$$
\begin{aligned}
\operatorname{dpll}(F) \Leftrightarrow & \operatorname{sat}(F[\text { selectLiteral }(F) \rightarrow \top]) \vee \\
& \operatorname{sat}(F[\text { selectLiteral }(F) \rightarrow \perp]) .
\end{aligned}
$$

Then, by Lemma 1, it holds that $\operatorname{dpll}(F) \Leftrightarrow \operatorname{sat}(F)$.

This proof, together with the termination argument, proves the total correctness of the dpll function. 


\section{Formalization in Isabelle/Isar}

Our formalization of the DPLL procedure and its correctness proof in Isabelle/Isar ${ }^{5}$ faithfully follow the definitions given in the previous sections. Using this formalization, an effective, operational ML implementation of the DPLL procedure is automatically generated from Isabelle, yielding a formally verified (although not quite efficient) SAT solver that is guaranteed to be correct (Haftmann, 2008). As an example, here we give some fragments of Isabelle/Isar code that formalizes some of the content given in the previous sections.
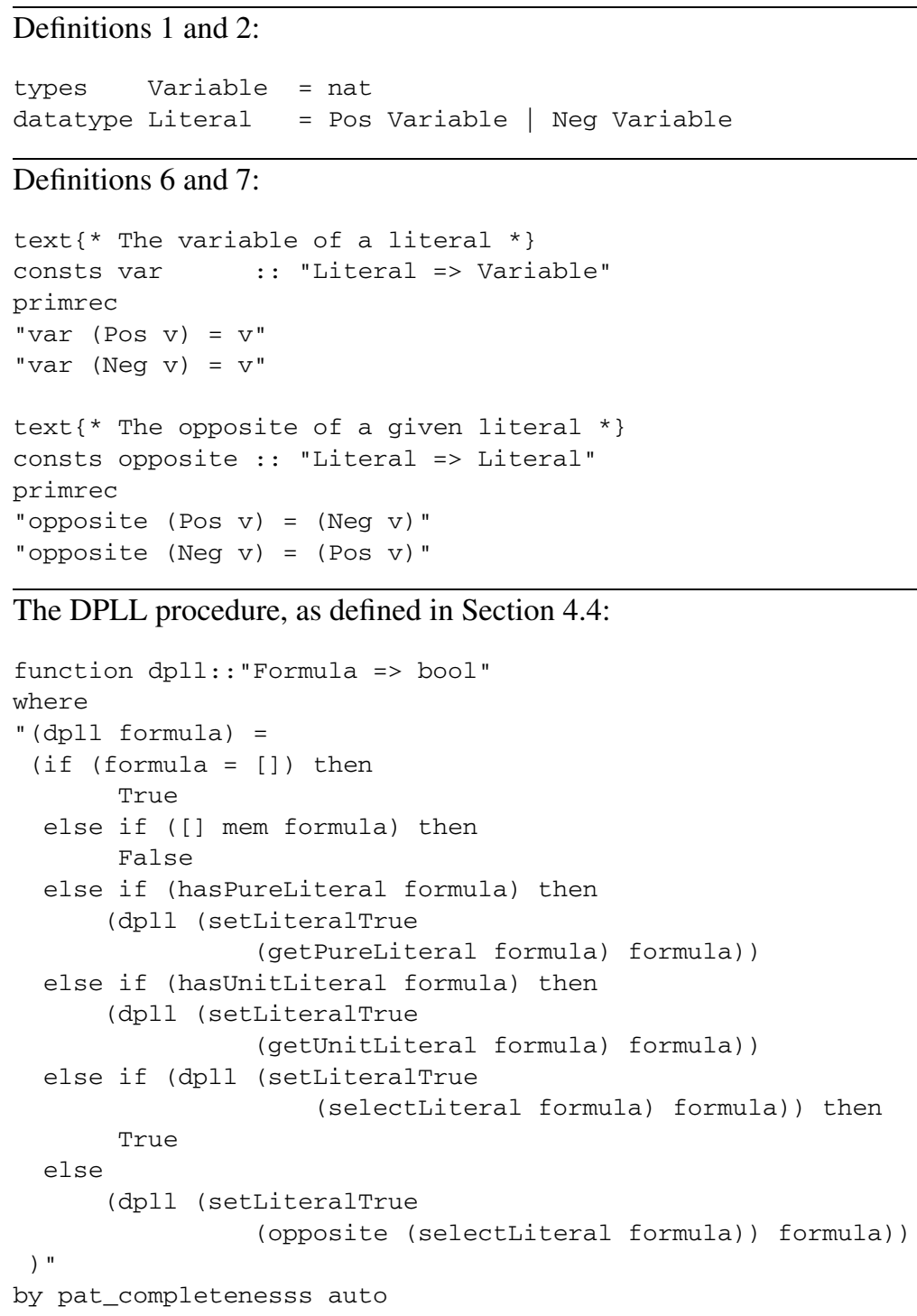

${ }^{5}$ All proof documents are available from $h t t p$ : / / argo . matf.bg.ac.rs. 
termination

by (relation "measure (\% formula. (numLiterals formula))")

(auto simp add: dpllTermination_1 dpllTermination_2

dpllTermination_3 dpllTermination_4)

The proof of correctness of the DPLL procedure, corresponding to the outlined proof given in Section 5:

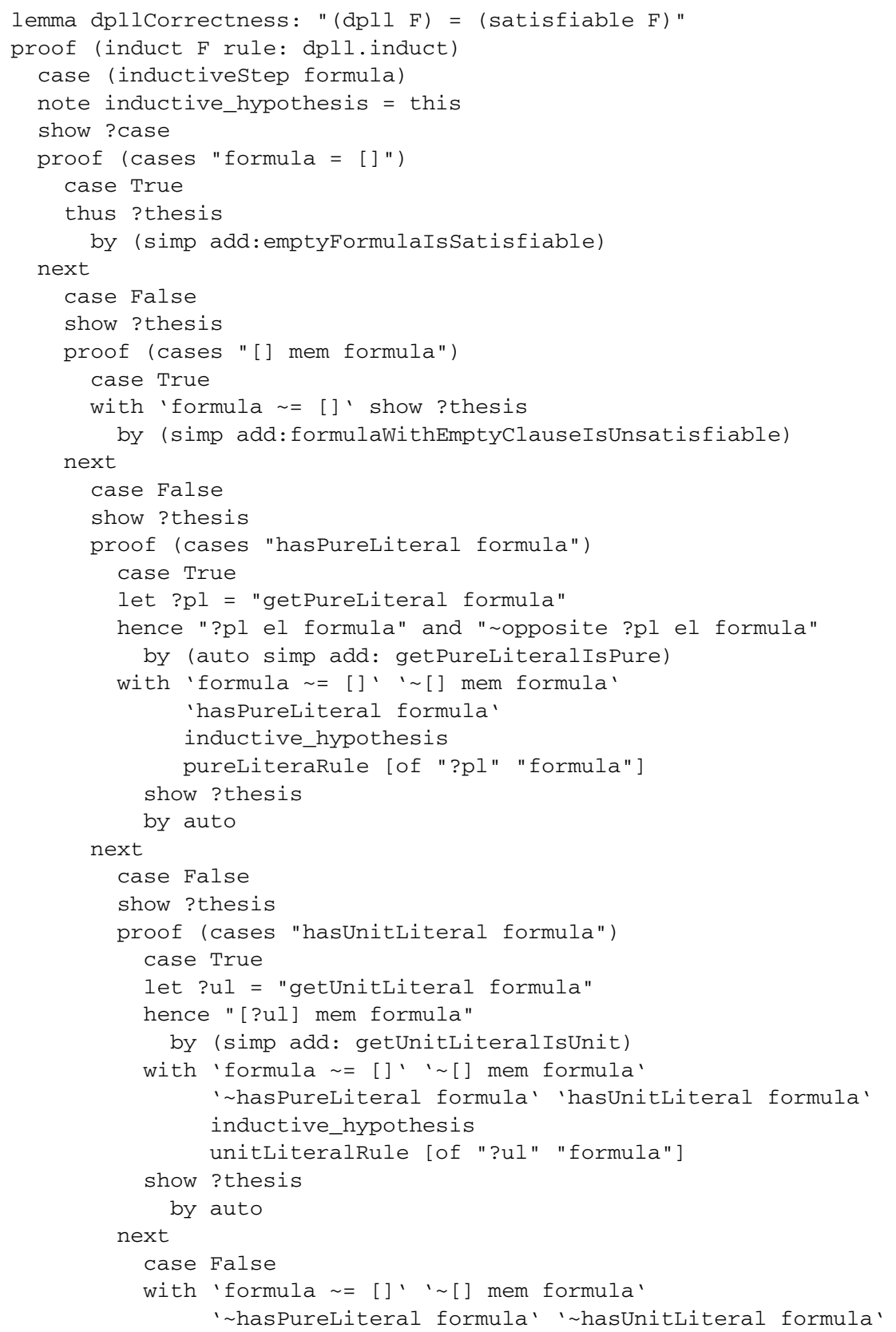




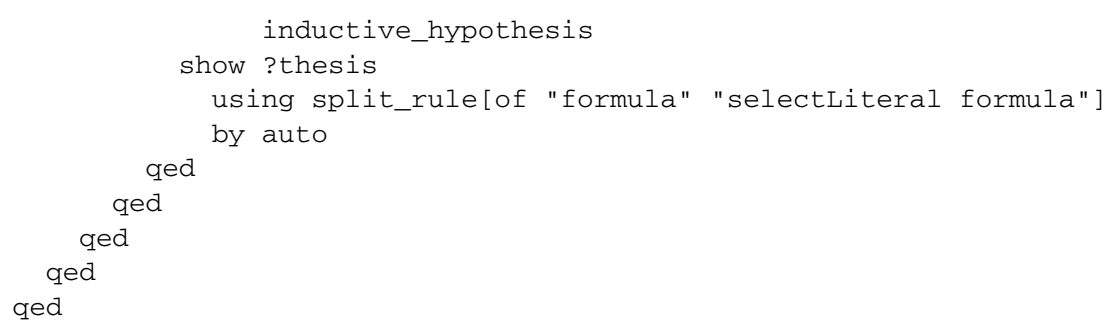

\section{Related Work}

There is a large and growing body of formalized mathematical knowledge. In this section we briefly overview formalized knowledge and proofs relevant for computer science, especially those formalized in Isabelle, and those relevant for automated reasoning and SAT and SMT solving.

Archive of formal proofs ${ }^{6}$ is a collection of proof libraries, examples, and larger scientific developments, mechanically checked in the theorem prover Isabelle. A range of algorithms and data structures have been formalized and verified in Isabelle and similar proof assistant tools. These algorithms include Quicksort, Binary Search, AVL Trees, Binary Search Trees, Depth First Search, Fast Fourier Transform, File Refinement, Cryptographic algorithms (Lindenberg et al., 2006), a range of distributed and parallel algorithms (Disk Paxos, Peterson's algorithm).

Flaws were detected in many security protocols (e.g., Li et al., 2007). Even if security protocols are accompained with correctness proofs, they can still be flawed if these proofs are not formally verifiable (e.g., Choo, 2006). Proof assistant tools have been used for formal verification of properties of various protocols (e.g., Nipkow, 2006; Barsotti et al., 2007).

A lot of efforts have been invested in verifying programming language semantics and compilers. For example, Klein and Nipkow introduced Jinja (Klein and Nipkow, 2006), a Java-like programming language with a formal semantics designed to exhibit core features of the Java language architecture. A model of the language, virtual machine and a compiler are then formally verified. Berghofer described a formally verified, fully executable compiler which was extracted from a proof assistant (Berghofer and Strecker, 2003). Blech and Glesner developed a formal semantics for static single assignment (SSA) phase of compilation (Blech and Glesner, 2004). Qian and Xu used iterative abstraction refinement and automated theorem proving for automatically verifying $\mathrm{C}$ programs against safety specifications (Qian and Xu, 2007).

Clark Barrett formally proved correctness of Stanford Framework for Cooperating Decision Procedures, but this proof, although quite detailed, was not verified using a proof assistant (Barret, 2003).

\footnotetext{
${ }^{6}$ http: //afp. sourceforge. net.
} 
Tom Ridge presented an efficient, mechanically verified sound and complete theorem prover for first order logic (Ridge, 2004). After formalization in Isabelle, OCaml code is generated, yielding a directly executable program.

Chaieb and Nipkow formalized and verified quantifier elimination based decision procedures for Presburger arithmetic (Chaieb and Nipkow, 2005).

Isabelle has been combined with different tools to achieve a higher degree of automation. For instance, Weber described integration of SAT solvers zChaff and MiniSat with Isabelle (Weber, 2005; Weber, 2006). Both SAT solvers generate resolution-style proofs of unsatisfiability of their input formulae. These proofs are verified by the theorem prover. Fontaine et al. (2006) used Isabelle to verify the correctness of proof traces generated by the SMT solver Harvey. Barsotti at al. experimented in combining the theorem prover Isabelle with automatic first-order arithmetic provers to increase automation on the verification of distributed protocols (Barsotti et al., 2007). As a case study for the experiment, they verified several clock synchronization algorithms.

Abstract descriptions of the DPLL algorithm and its extensions for ground Satisfiability Modulo Theory (SMT) have been developed. In Nieuwnhuis et al. (2006), Tinelli (2002), Krstić and Goel (2007), rule based presentations of these algorithms and their informal correctness proofs are given. Informal correctness proofs of the DPLL procedure can be found in many mathematical logic textbooks (Davis et al., 1994). However, as far as we know, our proof is the first formalized correctness proof for the DPLL procedure.

\section{Conclusions and Future Work}

In this paper we presented the first formal proof of correctness of the forty-five years old DPLL algorithm, one of the most fundamental algorithms in computer science. In its history, there were no doubts about the correctness of this algorithm. So, our proof does not resolve a long-standing mystery, but rather: (i) it adds to the growing body of formalized, verifiable mathematical knowledge, knowledge that can be verified by independent and reliable proof checkers; (ii) it serves as a first building block of formalized correctness proofs for modern SAT and SMT solvers. That task - formally proving correctness of state-of-the-art SAT and SMT solvers, very important for many applications, is in the focus of our current work.

\section{Appendix: One Concrete Implementation}

In this section we give very simple definitions of the functions used in the DPLL definition given in Section 4.4. They give a concrete, instantiated procedure and enable obtaining an effectively executable ML implementation. In order to have a more efficient SAT solver, these functions should be defined in a more sophisticated way.

A formula has a unit literal iff it has a clause with only one literal. We define hasUnitLiteral $(F)$ function by primitive recursion. 
DEFINITION 19.

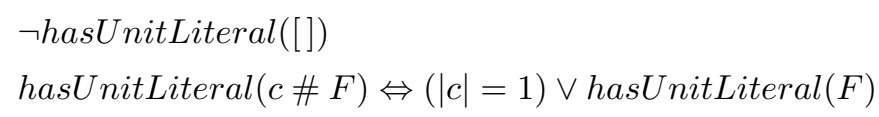

getUnitLiteral $(F)$ is the first literal $l$ such that $[l] \in F$. It is also defined by primitive recursion.

DEFINITION 20.

$$
\begin{aligned}
& \text { getUnitLiteral }(c \# F)= \\
& \text { if }|c|=1 \text { then } \\
& \text { head }(c) \\
& \text { else } \\
& \text { getUnitLiteral }(F)
\end{aligned}
$$

Procedures that find and select a pure literal from a formula are defined using a series of auxiliary functions.

The functions literals $(F)$ is a list that contains all literals that occur in the formula $F$. It is defined by primitive recursion.

DEFINITION 21.

$$
\begin{aligned}
& \operatorname{literals}([])=[] \\
& \operatorname{literals}(c \# F)=c @ \operatorname{literals}(F)
\end{aligned}
$$

The function hasPureLiteralAux $\left(c_{1}, c_{2}\right)$ checks if there is a literal from the list $c_{1}$ whose opposite literal does not occur in the list $c_{2}$. It is defined by primitive recursion.

\section{DEFINITION 22.}

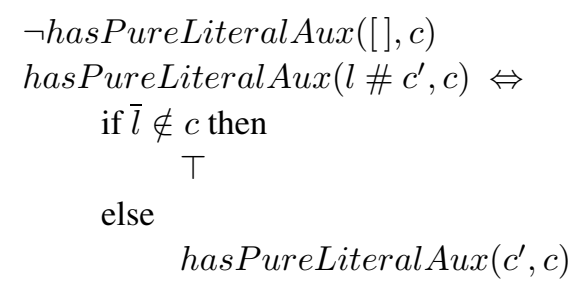

Using this auxiliary function, we define hasPureLiteral:

DEFINITION 23. hasPureLiteral $(F) \Leftrightarrow$ hasPureLiteralAux (literals $(F)$, literals $(F))$

The function getPureLiteralAux $\left(c_{1}, c_{2}\right)$ finds the literal from the list $c_{1}$ whose opposite literal does not occur in the list $c_{2}$. It is defined by primitive recursion. 
DEFINITION 24.

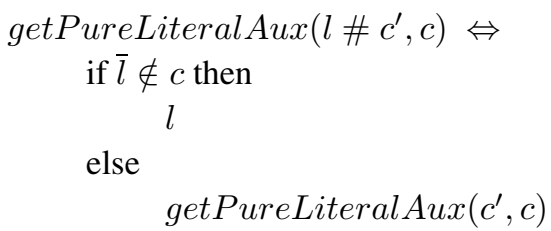

Finally, we can define the function getPureLiteral.

DEFINITION 25. getPureLiteral $(F)=\operatorname{getPureLiteralAux}($ literals $(F)$, literals $(F))$

selectLiteral $(F)$ is used to select an arbitrary literal of $F$. For example, it can be the first literal of the first clause of $F$.

DEFINITION 26. $\quad$ selectLiteral $(F)=\operatorname{head}(\operatorname{head}(F))$

It was proved that the functions defined in the above way meet the specification given in Section 4.4. These proofs can also be found in http: / / argo.matf.bg.ac.rs, while we don't present them here since this simple implementation is just one of many meeting the required specification.

Acknowledgments. We are grateful to Amine Chaieb and to the anonymous reviewers for useful comments on an earlier version of this paper.

\section{References}

Barrett, C. (2003). Checking Validity of Quantifier-Free Formulas in Combinations of First-Order Theories. $\mathrm{PhD}$ thesis, Stanford University.

Barsotti, D., Nieto, L.P., Tiu, A.F. (2007). Verification of clocksynchronization algorithms: Experiments on a combination of deductive tools. Formal Aspects of Computing, 19(3), 321-341.

Berghofer, S., Strecker, M. (2003). Extracting a formally verified, fully executable compiler from a proof assistant. Electronic Notes in Theoretical Computer Science, 82(2), 33-50.

Blech, J.O., Glesner, S. (2004). A formal correctness proof for code generation from SSA form in Isabelle/HOL. In: Jahrestagung der Gesellschaft für Informatik. LNI, Vol. 51. GI, pp. 449-458.

Chaieb, A., Nipkow, T. (2005). Verifying and reflecting quantifier elimination for presburger arithmetic. In: LPAR '05: Logic for Programming, Artificial Intelligence, and Reasoning. LNCS, Vol. 3835. Springer, pp. 367-380.

Choo, K.R. (2006). On the security analysis of Lee, Hwang \& Lee (2004) and Song \& Kim (2000) key exchange/agreement protocols. Informatica, 17(4), 467-480.

Cook, S.A. (1971). The complexity of theorem-proving procedures. In: STOC '71: Proceedings of the 3rd Annual ACM Aymposium on Theory of Computing. ACM, pp. 151-158.

Cook, S.A., Mitchell, D.G. (1997). Finding hard instances of the satisfiability problem: A survey. In: Satisfiability Problem: Theory and Applications. DIMACS Series in Discrete Mathematics and Theoretical Computer Science, Vol. 35. AMS, pp. 1-17.

Davis, M., Putnam, H. (1960). A computing procedure for quantification theory. Journal of the ACM, 7(3), 201-215.

Davis, M., Logemann, G., Loveland, D. (1962). A machine program for theorem-proving. Communications of the ACM, 5(7), 394-397. 
Davis, M., Sigal, R., Weyuker, E. (1994). Computability, Complexity, and Languages (Fundamentals of Theoretical Computer Science). Kaufmann/Academic Press.

Eén, N., Sörensson, N. (2003). An extensible SAT-solver. In: SAT '03: Theory and Applications of Satisfiability Testing. LNCS, Vol. 2919. Springer, pp. 502-518.

Fontaine, P., Marion, J.I., Merz, S., Nieto, L.P., Tiu, A.F. (2006). Expressiveness+Automation+Soundness: Towards combining, SMT solvers and interactive proof assistants. In: TACAS '06: Tools and Algorithms for the Construction and Analysis of Systems. LNCS, Vol. 3920, pp. 167-181.

Gu, J., Purdom, P.W., Franco, J., Wah, B. (1997). Algorithms for the satisfiability (SAT) problem: A survey. In: Satisfiability Problem: Theory and Applications. DIMACS Series in Descrete Mathematics and Theoretical Computer Science, Vol. 35. AMS, pp. 19-151.

Haftmann, F. (2008). Code Generation from Isabelle/HOL Theories. url: http://www.cl.cam.ac.uk/research/hvg/Isabelle/.

Irgens, M., Havens, W.S. (2004). On selection strategies for the DPLL algorithm. In: Advances in Artificial Intelligence. LNAI, Vol. 3060. Springer, pp. 277-291.

Klein, G., Nipkow, T. (2006). A machine-checked model for a Java-like language, virtual machine and compiler ACM Transactions on Programming Languages and Systems, 28(4), 619-695.

Krstić, S., Goel, A. (2007). Architecting solvers for SAT modulo theories: Nelson-Oppen with DPLL. In: FroCoS '07: Frontiers of Combining Systems. LNCS, Vol. 4720. Springer, pp. 1-27.

Le Berre, D., Simon, L. (Eds.) (2005). Special volume on the SAT 2005 competitions and evaluations. Journal on Satisfiability, Boolean Modeling and Computation, 2.

Li, C., Hwang, T., Lee, N. (2007). Security flaw in simple generalized group-oriented cryptosystem using ElGamal cryptosystem. Informatica, 18(1), 61-66.

Lindenberg, C., Wirt, K., Buchmann, J. (2006). Formal proof for the correctness of RSA-PSS. Cryptology ePrint Archive, Report 2006/011, http: //eprint. iacr.org/.

Moskewicz, M.W., Madigan, C.F., Zhao, Y., Zhang, L., Malik, S. (2001). Chaff: Engineering an efficient SAT solver. In: DAC '01: Proceedings of the 38th Conference on Design Automation. ACM, pp. 530-535.

Nieuwenhuis, R., Oliveras, A., Tinelli, C. (2006). Solving SAT and SAT modulo theories: From an abstract Davis-Putnam-Logemann-Loveland procedure to DPLL(T). Journal of the ACM, 53(6), 937-977.

Nipkow, T. (2006). Verifying a hotel key card system. In: ICTAC '06: Theoretical Aspects of Computing. LNCS, Vol. 4281, pp. 1-14.

Nipkow, T., Paulson, L.C., Wenzel, M. (2005). Isabelle HOL: A Proof Assistant for Higher-Order Logic. LNCS, Vol. 2283. Springer.

Paulson, L.C. (1994). Isabelle: A Generic Theorem Prover. LNCS, Vol. 828. Springer.

Qian, J., Xu, B. (2007). Formal verification for C program. Informatica, 18(2), 289-304.

Ranise, S., Tinelli, C. (2006). Satisfiability modulo theories. Trends and Controversies - IEEE Intelligent Systems Magazine, 21(6), 71-81.

Ridge, T. (2004). A mechanically verified, efficient, sound and complete theorem prover for first order logic. In: Archive of Formal Proofs. http: / / afp.sf . net.

Tinelli, C. (2002). A DPLL-based calculus for ground satisfiability modulo theories. In: JELIA '02: 8th European Conference on Logics in Artificial Intelligence. LNAI, Vol. 2424. Springer, pp. 308-319.

Weber, T. (2005). Using a SAT solver as a fast decision procedure for propositional logic in an LCF-style theorem prover. In: TPHOLs '05: Theorem Proving in Higher Order Logics (Emerging Trends). Research report PRG-RR-05-02. Computing Laboratory, Oxford University, pp. 180-189.

Weber, T. (2006). Efficiently checking propositional resolution proofs in Isabelle/HOL. In: Proceedings of the 6th International Workshop on the Implementation of Logics. CEUR Workshop Proceedings, Vol. 212. SUN Site Central Europe, pp. 44-62.

Wenzel, M. (2007). Isabelle/Isar - A generic framework for human-readable proof documents. In: From Insight to Proof: Studies in Logic, Grammar and Rethoric, Vol. 10(23). University of Bialystok, pp. 277-298.

Zhang, L., Malik, S. (2002). The quest for efficient boolean satisfiability solvers. In: CAV '02: Computer Aided Verification. LNCS, Vol. 2404. Springer, pp. 17-36. 
F. Marić is teaching assistant at the Faculty of Mathematics, University of Belgrade. He graduated in mathematics and received a master's degree in computer science from the University of Belgrade. His main research interests are in formal and automated theorem proving, SAT and SMT solving and object oriented programming.

P. Janičić is associate professor and a leader of the Automated Reasoning Group at the Faculty of Mathematics, University of Belgrade. He received his $\mathrm{PhD}$ degree in computer science from the University of Belgrade. His main research interests are in automated reasoning, especially in SAT and SMT solving and in intelligent geometrical software. 


\section{Formalus DPLL procedūros teisingumo įrodymas}

Filip MARIĆ, Predrag JANIČIĆ

Teiginiu logikos formuliu ivykdomumui tikrinti (žinomam kaip SAT problema) yra sukurti ivairūs efektyvūs algoritmai. Šie algoritmai yra vieno iš pirmuju algoritmu, skirtu ịvykdomumui tikrinti ir žinomo kaip Davis-Putnam-Logemann-Loveland (sutrumpintai, DPLL) procedūra, modifikacijos. DPLL turi daugybę taikymų, ịskaitant ir programinès bei techninès ịrangos verifikavimą. Daugelis moderniu SAT problemai spręsti skirtu programu remiasi šia procedūra, praplèsta ịvairiomis euristikomis. Straipsnyje pateikiamas formalus DPLL procedūros korektiškumo ịrodymas naudojant Isabelle/Isar sistemą. 